\title{
RESEARCH
}

Open Access

\section{KAT6A regulates stemness of aging bone marrow-derived mesenchymal stem cells through Nrf2/ARE signaling pathway}

Dongdong Fei ${ }^{1,2+}$, Yazheng Wang ${ }^{1 \dagger}$, Qiming Zhai ${ }^{2 \dagger}$, Xige Zhang ${ }^{1}$, Yang Zhang ${ }^{1}$, Yang Wang ${ }^{1}$, Bei $\mathrm{Li}^{2^{*}}$ and Qintao Wang ${ }^{1 *}$

\begin{abstract}
Background: This study aimed to explore the effect of KAT6A on the decreased stemness of aging bone marrowderived mesenchymal stem cells (BMSCs) and its potential mechanism.

Methods: The acetylation level and KAT6A expression of BMSCs from the young (YBMSCs) and the old (OBMSCs) were examined. Gain- and loss-of-function experiments were performed to determine the effect of KAT6A on BMSC proliferation, colony formation, and osteogenic differentiation. The effect of KAT6A on Nrf2/ARE signaling pathway was investigated after KAT6A inhibition in YBMSCs or overexpression in OBMSCs, and the role of Nrf2/ARE signaling pathway on stemness was examined by investigating proliferation, colony formation, and osteogenic differentiation. Further in vivo study was performed to explore osteogenesis ability of OBMSCs after modulation of KAT6A and Nrf2/ARE pathway through cell sheet technology.

Results: The acetylation level and KAT6A expression of OBMSCs were decreased, and KAT6A downregulation resulted in decreased proliferation, colony formation, and osteogenic differentiation of OBMSCs. Mechanically, KAT6A was found to regulate Nrf2/ARE signaling pathway and inhibit ROS accumulation in OBMSCs, thus promoting proliferation, colony formation, and osteogenic differentiation of OBMSCs. Further study demonstrated that KAT6A could promote osteogenesis of OBMSCs by regulating Nrf2/ARE signaling pathway.

Conclusions: Downregulation of KAT6A resulted in the decreased stemness of OBMSCs by inhibiting the Nrf2/ARE signaling pathway.
\end{abstract}

Keywords: KAT6A, Nrf2/ARE signaling pathway, Aging, Bone marrow-derived mesenchymal stem cells, Stemness

\footnotetext{
* Correspondence: Ibfmmu@163.com; yznmbk@fmmu.edu.cn

${ }^{\dagger}$ Dongdong Fei, Yazheng Wang and Qiming Zhai contributed equally to this work.

${ }^{2}$ State Key Laboratory of Military Stomatology \& National Clinical Research Center for Oral Diseases \& Shaanxi International Joint Research Center for Oral Diseases, Center for Tissue Engineering, School of Stomatology, The Fourth Military Medical University, Xi'an 710032, Shaanxi, China

'State Key Laboratory of Military Stomatology \& National Clinical Research Center for Oral Diseases \& Shaanxi Engineering Research Center for Dental Materials and Advanced Manufacture, Department of Periodontology, School of Stomatology, The Fourth Military Medical University, Xi'an 710032, Shaanxi, China
}

C C The Author(s). 2021 Open Access This article is licensed under a Creative Commons Attribution 4.0 International License, which permits use, sharing, adaptation, distribution and reproduction in any medium or format, as long as you give appropriate credit to the original author(s) and the source, provide a link to the Creative Commons licence, and indicate if changes were made. The images or other third party material in this article are included in the article's Creative Commons licence, unless indicated otherwise in a credit line to the material. If material is not included in the article's Creative Commons licence and your intended use is not permitted by statutory regulation or exceeds the permitted use, you will need to obtain permission directly from the copyright holder. To view a copy of this licence, visit http://creativecommons.org/licenses/by/4.0/. The Creative Commons Public Domain Dedication waiver (http://creativecommons.org/publicdomain/zero/1.0/) applies to the data made available in this article, unless otherwise stated in a credit line to the data. 


\section{Background}

Age-related osteoporosis, characterized by bone fragility, has become one of the main risks that endanger the health of the elderly. Bone marrow-derived mesenchymal stem cells (BMSCs) are essential for bone formation, and decreased stemness of BMSCs has been regarded as one important factor contributing to age-related osteoporosis $[1,2]$. Numerous studies have shed light on the mechanism of decreased stemness of BMSCs during aging; however, how aging leads to this still needs further investigation.

Epigenetic alteration, including changes of methylation and acetylation of DNA and histones, has been regarded as one hallmark of aging through changing genomic instability and gene expression profiles [3, 4]. Histone acetyltransferases (HATs), such as KAT2A, elongator complex protein 3, and KAT6A, are enzymes responsible for histone lysine residues acetylation. They have numerous functions including DNA repair and transcriptional regulation [5]. Several studies have also shown that HATs can regulate stemness of MSCs [6-8]. The KAT6A gene, which can acetylate lysine residues in histone $\mathrm{H} 3$, is required for hematopoiesis [9] and skeletogenesis, as well as the regulation of many genes. Previous study has indicated that KAT6A could act as a transcriptional coactivator for RUNX family transcription factor 2 (Runx2), and dysregulation of KAT6A can lead to impaired skeletogenesis. Besides, KAT6A was also found to regulate cellular senescence [10-12]. Several studies reported that KAT6A could regulate the self-renewal, proliferation, and differentiation of hematopoietic stem cells [9]; however, whether and how KAT6A regulated the decreased stemness of aging BMSCs remains unknown.

Oxidative stress, a situation that occurs when the accumulation of reactive oxygen species (ROS) overwhelms the antioxidant capacity, is related to the development of several diseases and has become an important predisposing factor for stem cell aging and age-related diseases [1315]. When confronted with oxidative stress, transcription factor nuclear factor erythroid 2-related factor 2 (Nrf2) can accumulate in the nucleus and bind to the antioxidant response element to promote the expressions of antioxidant enzymes to maintain a stable internal environment [16]. Studies confirmed that decreased Nrf2/ARE signaling pathway can lead to stem cell dysfunction $[17,18]$, but the detailed mechanism needs further investigation. Some studies have shown that HATs can regulate Nrf2/ARE signaling pathway $[19,20]$, and one study reported that MOF, a HAT belonging to the MYST family, could promote nuclear retention of Nrf2 [21]. Similar to MOF, KAT6A also belongs to the MYST family and shares some structural similarities with MOF, indicating that KAT6A may also be implicated in the regulation of Nrf2/ARE signaling pathway. In our study, decreased KAT6A expression in OBMSCs was found, and downregulated KAT6A expression was demonstrated to contribute to the decreased stemness of OBMSCs. Mechanically, KAT6A was found to scavenge accumulated ROS in OBMSCs through the Nrf2/ARE signaling pathway, thus ameliorating stemness and improving osteogenesis of OBMSCs.

\section{Methods \\ Cell culture}

Human BMSCs collected from the First Hospital Affiliated to the Fourth Military Medical University were used in this study with the approval of the Ethics Committee of the Fourth Military Medical University. YBMSCs were from persons who are under 30 years old, and OBMSCs were from those who are over 55 years old. To isolate human BMSCs, bone marrow samples from the iliac were diluted using Dulbecco's phosphate-buffered saline, followed by centrifugation for $30 \mathrm{~min}$ at a speed of 2000 $\mathrm{rpm}$. Then, the cells were plated in the $10-\mathrm{cm}$ dish and were cultured in the $\alpha$-MEM supplemented with $10 \%$ fetal bovine serum (FBS; Thermo Electron, Melbourne, Australia), $100 \mathrm{U} / \mathrm{ml}$ penicillin, $100 \mathrm{mg} / \mathrm{ml}$ streptomycin, and $0.292 \mathrm{mg} / \mathrm{ml}$ glutamine (Invitrogen, Carlsbad, CA, USA). BMSCs at passages 3-6 were used, and the medium was refreshed every 3 days. ML 385 (R \& D Systems, 6243, MI, USA), a specific Nrf2 inhibitor, was dissolved in DMSO. For the culture of BMSC sheets, $1 \times 10^{6}$ cells were seeded on six-well dishes and cell sheet-induction medium $(50 \mu \mathrm{g} / \mathrm{ml}$ vitamin $\mathrm{C})$ was added to promote cell sheet formation after being cultured for $24 \mathrm{~h}$. After 14 days, BMSC sheets were collected for animal study.

\section{Colony-forming unit-fibroblastic (CFU-F) assay}

BMSCs treated at a density of $2 \times 10^{3}$ were seeded in a 5 -cm dish, and the medium was refreshed every 3 days. After 12 days of culture, the dish was washed using PBS twice and was fixed with $4 \%$ paraformaldehyde (SigmaAldrich Corp, St Louis, MO, USA) for $30 \mathrm{~min}$. Then, 2\% crystal violet (Amresco, Solon, OH, USA) was used to stain the clones. Clone that contains more than 50 cells under the stereomicroscope was defined as a colony.

\section{Cell proliferation assay}

BMSCs were seeded in 96-well plates at a density of $2 \times$ 10 [3]. After 12-24 h, $10 \mu \mathrm{l}$ enhanced Cell Counting Kit8 (CCK-8) (Beyotime, China) was added to each well and cells were transfected with corresponding reagents. The absorbance values at day 1 , day 3 , day 5 , and day 7 were measured at $450 \mathrm{~nm}$ using a microplate reader.

\section{Osteogenic differentiation assay}

BMSCs treated at a density of $2 \times 10^{5}$ were plated on 12 well dishes and were cultured in the $\alpha$-MEM until 80 90\% confluence. BMSCs were then cultured under 
an osteogenic culture medium supplemented with $100 \mu \mathrm{g} / \mathrm{ml}$ ascorbic acid (Sigma, USA), $10 \mathrm{nmol} / \mathrm{l}$ dexamethasone (Sigma, USA), and $5 \mathrm{mmol} / \mathrm{l} \beta$ glycerophosphate (Sigma, USA). After osteogenic induction for 14 days, quantitative reverse transcription polymerase chain reaction (qRT-PCR) was performed to explore the expressions of runt-related transcription factor 2 (Runx2), osteocalcin (OCN), and bone morphogenetic protein 2 (BMP2). After osteogenic induction for 21 days, calcium deposit was assessed using alizarin red staining, and $10 \%$ cetylpyridinium chloride was used for quantitative analysis at 562-nm absorbance value.

\section{Transfection assay}

BMSCs treated at a density of $2 \times 10^{5}$ were plated on 12 well dishes until 80 90\% confluence followed by serum starvation for $2 \mathrm{~h}$. For siRNA experiments, BMSCs at the experiment groups were transfected with a final concentration of $50 \mathrm{nM}$ KAT6A siRNA (Ribobio, Guangzhou, China) or Nrf2 siRNA (Santa Cruz Biotechnology, sc37030, Texas, USA), with scrambled siRNA (Santa Cruz Biotechnology, sc-37007, USA) at the control groups. For overexpression experiments, BMSCs at the experiment groups were transfected with $500 \mathrm{ng}$ plasmid of KAT6A (OBiO Technology, Shanghai, China), with control overexpression vectors at the control groups. The transfection reagent Lipofectamine 2000 (Invitrogen, USA) was used as per the manufacturer's instructions. After transfection, the culture medium was replaced by growth culture medium and BMSCs were harvested at $48 \mathrm{~h}$ for RNA and $72 \mathrm{~h}$ for protein detection. For osteogenic differentiation assay, the osteogenic induction medium was used in the following day.

\section{Quantitative RT-PCR}

TRIzol Reagent TM (Invitrogen, USA) was used to extract total cellular RNA as per the manufacturer's instructions, and cDNA was synthesized in a $20-\mu$ l reaction volume. The qRT-PCR reactions were performed using the SYBR Premix Ex TaqTMII kit (Takara, Tokyo, Japan), and CFX96 Touch Real-Time System (Bio-Rad, CA, USA) was used for detection. Fold changes of mRNA were calculated using the $2^{-\Delta \Delta \mathrm{Ct}}$ method with the reference gene glyceraldehyde-3-phosphate dehydrogenase (GAPDH). The primer sequences are listed in Supplementary Table $\mathrm{S} 1$ in Additional file 1.

\section{Western blot analysis}

Cells were lysed in RIPA lysis buffer (Beyotime Institute of Biotechnology, Shanghai, China) after being washed with PBS twice. To prepare the cytoplasmic component and nuclear component, BMSCs were lysed using nuclear and cytoplasmic protein extraction kit (Beyotime Institute of Biotechnology, China) as per manufacturer's instructions. After separation and transferring using 10\% Tris-glycine SDS-polyacrylamide gels and PVDF membranes, samples were blocked using $5 \%$ bovine serum albumin for $2 \mathrm{~h}$. Then, PVDF membranes were incubated overnight at $4{ }^{\circ} \mathrm{C}$ with the following primary antibodies: anti-GAPDH (CWBIO, CW0100, Beijing, China), anti- $\beta$ tubulin (CWBIO, CW0098, China), anti-KAT6A (Santa Cruz Biotechnology, sc-5713, USA), anti-acetylatedlysine (Cell Signaling Technology, \#9441, Beverly, MA, USA), anti-Histone H3 (Cell Signaling Technology, \#9715, USA), anti-acetyl-Histone H3 (Millipore, 17-615, Billerica, CA, USA), anti-Histone H4 (Abcam, ab177840, Cambridge, UK), anti-acetyl-Histone H4 (R \& D Systems, AF5215-SP, USA), anti-Histone H3 (acetyl K9) (Abcam, ab10812, UK), anti-Histone H3 (acetyl K14) (Abcam, ab52946, UK), and anti-Nrf2 (Cell Signaling Technology, \#12721, USA). After incubation with secondary antibody (Jackson, West Grove, PA, USA) for $2 \mathrm{~h}$ at $37^{\circ} \mathrm{C}$, membranes were visualized using the enhanced chemiluminescence kit (Pierce, IL, USA). ImageJ (Media Cybernetics, MD, USA) was used for quantitative analysis.

\section{ROS detection}

BMSCs were seeded in 96-well plates at a density of $2 \times$ $10^{4}$ and were cultured using $\alpha$-MEM supplemented with $10 \% \mathrm{FBS}$ and $0.292 \mathrm{mg} / \mathrm{ml}$ glutamine. After $12-24 \mathrm{~h}$, cells were transfected with corresponding reagents. In detail, for siRNA experiments, BMSCs were transfected with KAT6A siRNA, Nrf2 siRNA, or scrambled siRNA with a final concentration of $50 \mathrm{nM}$. For overexpression experiments, BMSCs were transfected with $50 \mathrm{ng}$ plasmid of KAT6A or control overexpression vectors. For studies involving transfection of siRNA and plasmid, $50 \mathrm{nM}$ siRNA and $50 \mathrm{ng}$ plasmid were mixed and transfected at the same time. After transfection for $24 \mathrm{~h}$, the medium was changed using $100 \mu \mathrm{l}$ ROS red working solution (Abcam, ab186027, UK). After incubation at $37^{\circ} \mathrm{C}$ for $60 \mathrm{~min}$, a fluorescence microplate reader (PerkinElmer, MA, USA) was used to detect the intensity of fluorescence at 520-nm and 605-nm excitation and emission wavelengths, respectively.

\section{Animal study}

The animal study was conducted according to the committee guidelines of the Animal Care Committee of the Fourth Military Medical University. Eight-week-old Sprague-Dawley (SD) male rats were randomly assigned to three groups with each group containing seven or eight rats. The surgical procedure of the alveolar bone defects was described as previously mentioned [22]. Briefly, SD rats were anesthetized by intraperitoneal administration of $1 \%$ pentobarbital sodium solution. After being shaved by a razor and having disinfected with 
iodophor, the entire skin along the lower edge of the unilateral mandible was cut. Then, the masseter muscle was cut off to expose the buccal side of the mandible, and a dental handpiece was used to remove the alveolar bone between the first and second molars about 1.0 to $1.5 \mathrm{~mm}$ away from the top of the alveolar ridge to make a periodontal defect with $3 \mathrm{~mm}$ long, $2 \mathrm{~mm}$ wide, and 1 $\mathrm{mm}$ thickness. BMSC sheets (OBMSCs + DMSO + vector, OBMSCs + DMSO + KAT6A plasmid, and OBMSCs $+10 \mu \mathrm{m}$ ML $385+$ KAT6A plasmid) were then filled into the defects. After 6 weeks, the SD rats were euthanized and the samples were collected for micro-CT analysis.

\section{Statistical analyses}

All data are displayed as mean $\pm \mathrm{SD}$. Comparisons between the two groups were performed using two-tailed Student's $t$ test, and comparisons among the three groups were performed using one-way ANOVA. SPSS13.0 or GraphPad 5 was used, and significance was confirmed at $P<0.05$ ( $P<0.05$; ${ }^{* *} P<0.01$; $\left.{ }^{* * *} P<0.001\right)$.

\section{Results}

\section{The KAT6A expressions were decreased in OBMSCs}

Firstly, the stemness of YBMSCs and OBMSCs was investigated. CCK-8 and crystal violet analysis found lower proliferative and self-renewal capacity of OBMSCs than that of YBMSCs (Figure S1A and S1B). Besides, qRTPCR and alizarin red staining confirmed the decreased osteogenesis ability of OBMSCs than that of YBMSCs (Figure S1C and S1D). These results indicated that OBMSCs exhibited lower stemness than YBMSCs. For more details, please refer to Additional file 2 .

Then, the levels of acetylated lysine in YBMSCs and OBMSCs were explored, and the acetylation levels of lysine were found to be decreased in OBMSCs compared to those in YBMSCs (Fig. 1a), indicating that histone acetylation may be involved in the decreased stemness of OBMSCs. Moreover, histone $\mathrm{H} 3$ and histone $\mathrm{H} 4$ acetylation levels were examined, and western blot analysis showed decreased histone $\mathrm{H} 3$ acetylation levels in OBMSCs, while no significant difference was found in histone H4 acetylation levels (Fig. 1b). KAT6A is a HAT that is specific for acetylation of histone H3. The mRNA expressions of KAT6A in YBMSCs and OBMSCs were investigated, and qRT-PCR results showed that KAT6A mRNA expressions were downregulated in OBMSCs compared to that in YBMSCs (Fig. 1c). Downregulated KAT6A expressions in OBMSCs were also confirmed by Western blot (Fig. 1d). To further confirm the effect of decreased KAT6A expressions on OBMSCs, KAT6Aspecific acetylation sites H3K9 [10] and H3K14 [23] were examined, and decreased acetyl-H3K9 and acetylH3K14 in OBMSCs were found (Fig. 1e, f). All these results proved that KAT6A expressions were decreased in OBMSCs.

\section{KAT6A regulated stemness of BMSCs}

To demonstrate whether KAT6A regulated stemness of BMSCs, KAT6A expression in YBMSCs was downregulated, and KAT6A expression in OBMSCs was upregulated through KAT6A siRNA and overexpression plasmid. Transfection efficiencies of inhibition and overexpression were confirmed using qRT-PCR (Fig. 2a, f). After KAT6A inhibition, the proliferative capacity of KAT6A-inhibited YBMSCs was lower than that of the scramble-treated YBMSCs (Fig. 2b). Additionally, the colony-forming ability of YBMSCs was also decreased after KAT6A inhibition (Fig. 2c). Besides, osteogenicrelated genes of Runx2, OCN, and BMP2 were found to be downregulated after KAT6A inhibition in YBMSCs as confirmed by qRT-PCR (Fig. 2d). Alizarin red staining further confirmed decreased mineral node formation in KAT6A-inhibited YBMSCs (Fig. 2e). Conversely, KAT6A overexpression could improve the proliferative capacity and the colony-forming ability of OBMSCs (Fig. 2g, h). Besides, KAT6A overexpression could increase the expressions of osteogenic-related genes of Runx2, OCN, and $B M P 2$ (Fig. 2i) and promote more mineral node formation of OBMSCs (Fig. 2j).

\section{Decreased KAT6A expression led to ROS accumulation in OBMSCs by inhibiting Nrf2/ARE signaling pathway}

The accumulation of ROS during the aging process can contribute to stem cell dysfunction, and Nrf2/ ARE signaling pathway is responsible for ROS scavenging. Several studies demonstrated that HATs could regulate Nrf2/ARE signaling pathway. Thus, KAT6A was suspected to improve stemness of BMSCs through Nrf2/ARE signaling pathway. In this study, accumulated ROS were found in OBMSCs (Fig. 3e), and intranuclear Nrf2 level in OBMSCs was downregulated as confirmed by western blot (Fig. 3a). Moreover, the target genes of Nrf2/ARE signaling pathway, glutamate-cysteine ligase catalytic subunit (GCLC) and $N A D(P) H$ quinone dehydrogenase 1 (NQO1), were also downregulated in OBMSCs (Fig. 3d). All these results indicated that Nrf2/ARE signaling pathway was inhibited during the aging process. Then, KAT6A was inhibited in YBMSCs and KAT6A was overexpressed in OBMSCs, and the results showed that decreased KAT6A expression in OBMSCs could result in ROS accumulation, as KAT6A-inhibited YBMSCs presented more ROS accumulation and KAT6A-overexpressed OBMSCs presented less ROS accumulation (Fig. 3g, i). Meanwhile, less intranuclear Nrf2 expressions were detected after KAT6A inhibition in YBMSCs (Fig. 3b), while more intranuclear Nrf2 expressions were 

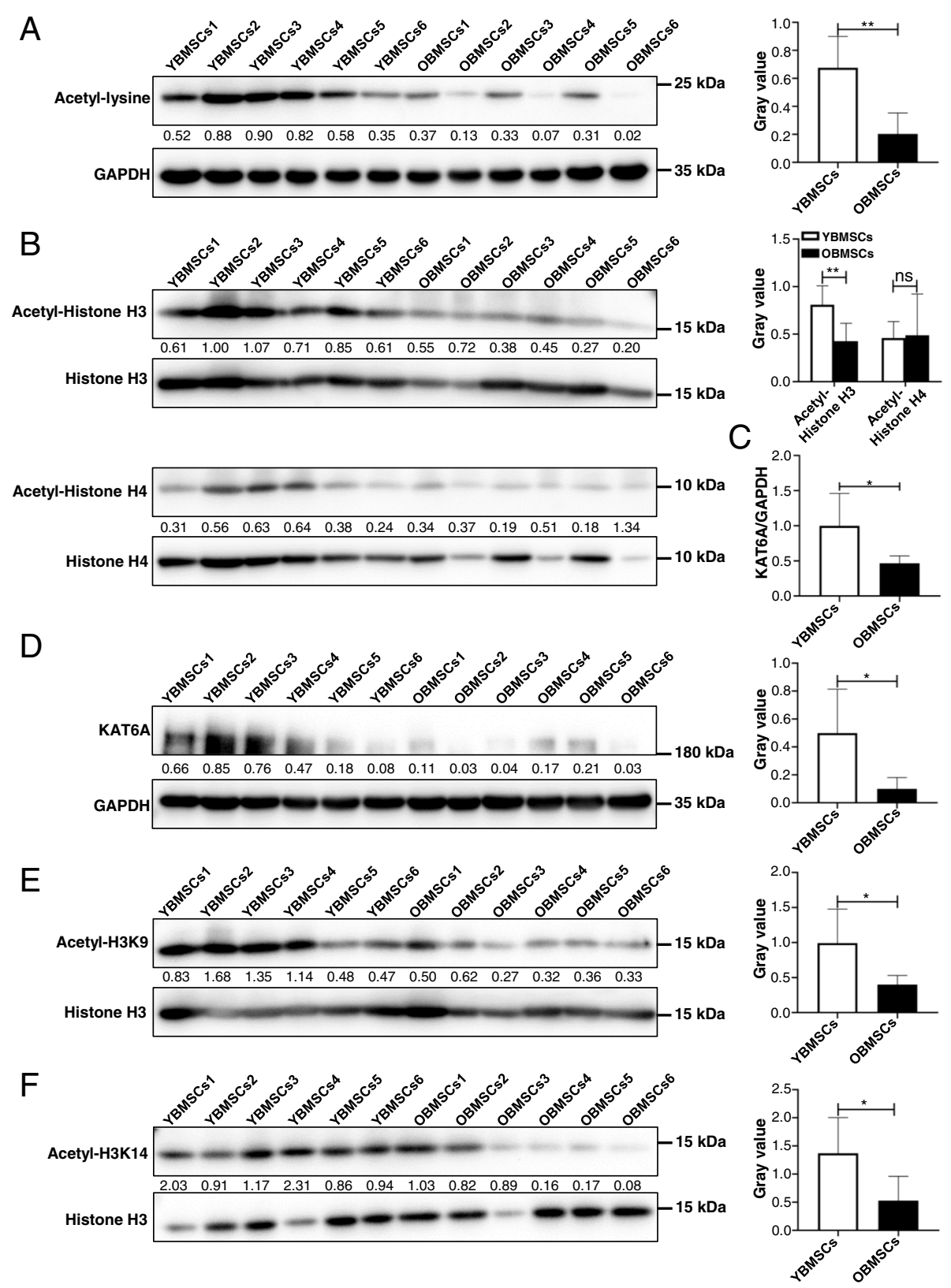

Fig. 1 The KAT6A expressions were decreased in aging BMSCs. a The acetylation levels of lysine in YBMSCs and OBMSCs were explored by western blot, and quantitative analysis was performed by ImageJ $(n=6)$. b The expressions of Histone H3, acetyl-Histone H3, Histone H4, and acetyl-Histone H4 were explored by western blot, and quantitative analysis was performed by ImageJ $(n=6)$. $\mathbf{c}$ The expressions of KAT6A mRNA in YBMSCs and OBMSCs were explored by qRT-PCR $(n=6)$. $\mathbf{d}$ The expressions of KAT6A protein in YBMSCs and OBMSCs were explored by western blot, and quantitative analysis was performed by ImageJ $(n=6)$. e The acetylation levels of H3K9 in YBMSCs and OBMSCs were explored by western blot, and quantitative analysis was performed by ImageJ $(n=6)$. f The acetylation levels of H3K14 in YBMSCs and OBMSCs were explored by western blot, and quantitative analysis was performed by ImageJ $(n=6)$

detected after KAT6A overexpression in OBMSCs (Fig. 3c). Besides, the target genes of Nrf2/ARE signaling pathway were also downregulated in KAT6Ainhibited YBMSCs and were upregulated in KAT6Aoverexpressed OBMSCs (Fig. 3f, h). These results supported that KAT6A could regulate Nrf2/ARE signaling pathway.
KAT6A regulated stemness of OBMSCs through Nrf2/ARE signaling pathway

To demonstrate that KAT6A regulated stemness of OBMSCs through Nrf2/ARE signaling pathway, first, Nrf2/ARE signaling pathway was inhibited to explore the relationship between the Nrf2/ARE signaling pathway and the decreased stemness of OBMSCs. Nrf2 


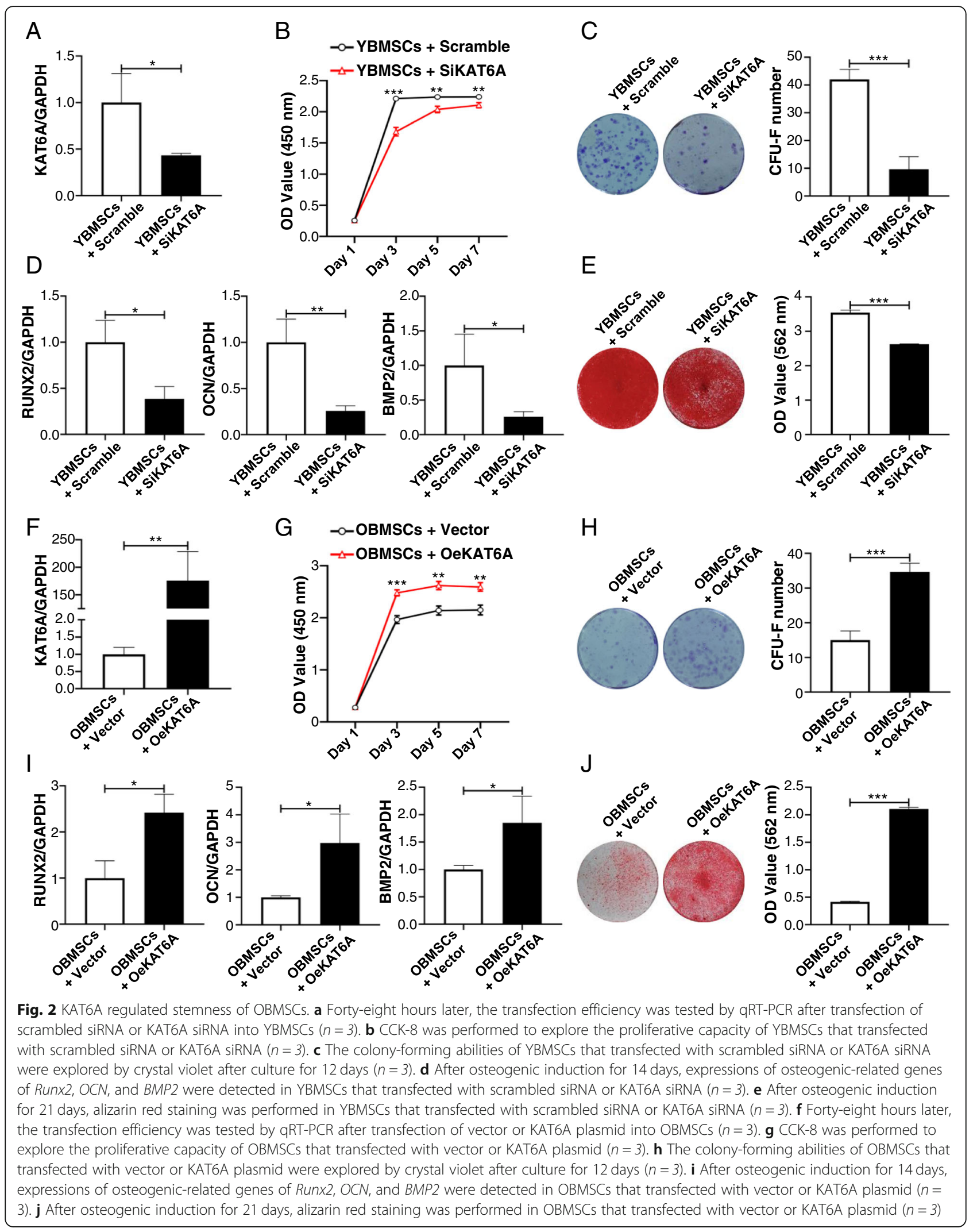




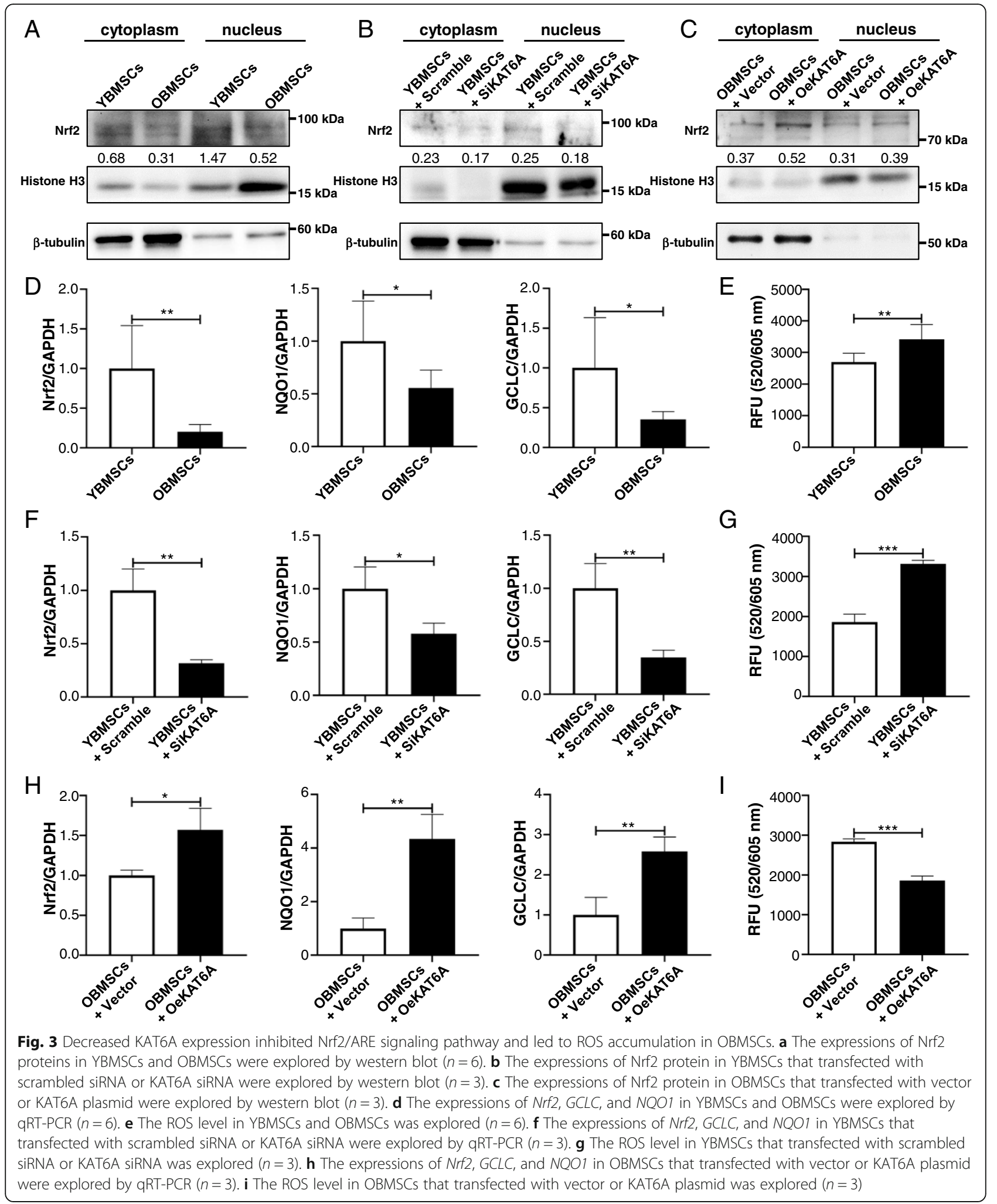

siRNA was used to inhibit Nrf2/ARE signaling pathway of YBMSCs (Fig. 4a), and the results showed that the inhibition of Nrf2/ARE signaling pathway could increase
ROS level in YBMSCs (Fig. 4b). After Nrf2 inhibition, the proliferative capacity of YBMSCs was decreased (Fig. 4c), and the colony-forming ability was also 

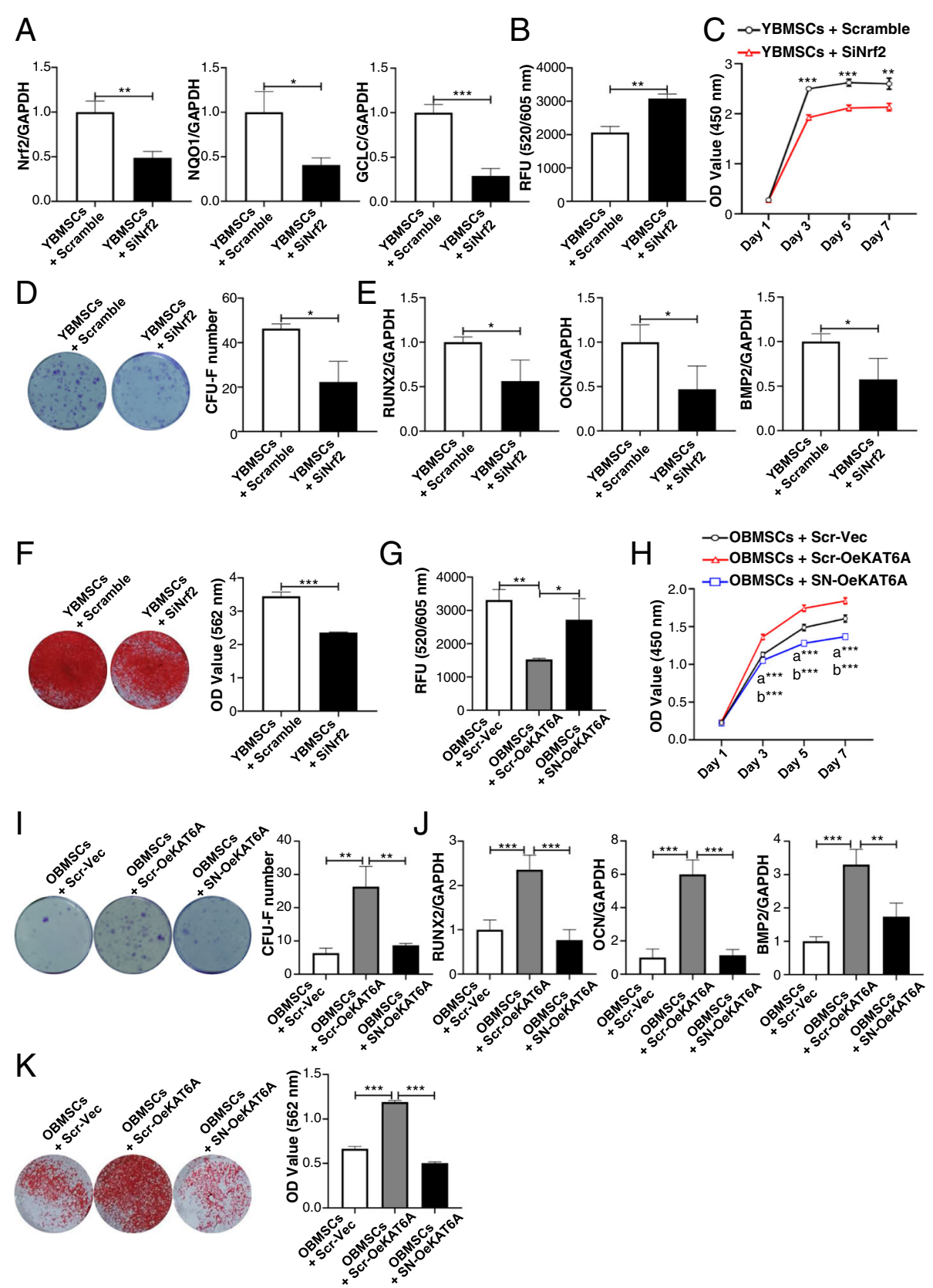

Fig. 4 KAT6A regulated stemness of OBMSCS through Nrf2/ARE signaling pathway. a Forty-eight hours later, the expressions of Nrf2, GCLC, and NQO1 in YBMSCS that transfected with scrambled siRNA or Nrf2 siRNA were explored by qRT-PCR $(n=3)$. b The ROS level in YBMSCs that transfected with scrambled siRNA or Nrf2 siRNA was explored ( $n=3)$. $\mathbf{c}$ CCK-8 was performed to explore the proliferative capacity of YBMSCs that transfected with scrambled siRNA or Nrf2 siRNA ( $n=3)$. $\mathbf{d}$ The colony-forming abilities of YBMSCs that transfected with scrambled siRNA or Nrf2 siRNA were explored by crystal violet after culture for 12 days $(n=3)$. e After osteogenic induction for 14 days, expressions of osteogenic-related genes of Runx2, OCN, and BMP2 were detected in YBMSCs that transfected with scrambled siRNA or Nrf2 siRNA $(n=3)$. f After osteogenic induction for 21 days, alizarin red staining was performed in YBMSCs that transfected with scrambled siRNA or Nrf2 siRNA $(n=3)$. $\mathbf{g}$ After overexpressing KAT6A in OBMSCs in the content of Nrf2 siRNA, the ROS level was explored $(n=3)$. $\mathbf{h}$ After overexpressing KAT6A in OBMSCS in the content of Nrf2 siRNA, CCK-8 was performed to explore the proliferative capacity $(n=3)$. i After overexpressing KAT6A in OBMSCs in the content of Nrf2 siRNA, the colony-forming abilities were explored by crystal violet after culture for 12 days $(n=3)$. $\mathbf{j}$ After overexpressing KAT6A in OBMSCs in the content of Nrf2 siRNA, expressions of osteogenic-related genes of Runx2, OCN, and BMP2 were detected $(n=3)$. $\mathbf{k}$ After overexpressing KAT6A in OBMSCs in the content of Nrf2 siRNA, alizarin red staining was performed $(n=3)$. Scr-Vec, scramble + vector; Scr-OeKAT6A, scramble + OeKAT6A; SN-OeKAT6A, SiNrf2 + OeKAT6A; a "OBMSCs + Scr-Vec" compared with "OBMSCS + Scr-OeKAT6A"; b "OBMSCs + Scr-OeKAT6A" compared with "OBMSCS + SN-OeKAT6A" 
downregulated (Fig. 4d). Besides, results from qRT-PCR and alizarin red staining showed that the osteogenic differentiation of YBMSCs was inhibited after Nrf2 inhibition (Fig. 4e, f). To further demonstrate that Nrf2/ARE signaling pathway was involved in KAT6A-mediated improvement of stemness of OBMSCs, KAT6A was also overexpressed in OBMSCs in the content of Nrf2 siRNA, and ROS level was increased after Nrf2 inhibition in KAT6A-overexpressed OBMSCs (Fig. 4g). The proliferative capacity and the colony-forming ability were also inhibited after Nrf2 inhibition (Fig. 4h, i). Meanwhile, qRT-PCR and alizarin red staining analysis showed that KAT6A regulated osteogenic differentiation of aging BMSCs through Nrf2/ARE signaling pathway (Fig. 4j, k).

\section{KAT6A regulated osteogenesis of aging BMSCs through Nrf2/ARE signaling pathway in vivo}

In vivo study was also performed to demonstrate the osteogenesis of BMSCs after KAT6A and/or Nrf2 modification. ML 385, a specific Nrf2 inhibitor, was used to inhibit Nrf2/ARE signaling pathway, and the concentration of ML 385 was chosen according to previous researches [24, 25]. Then, OBMSCs were treated with "DMSO + vector" or "DMSO + KAT6A plasmid" or KAT6A plasmid with $1 \mu \mathrm{M}, 5 \mu \mathrm{M}, 10 \mu \mathrm{M}$, or $20 \mu \mathrm{M} \mathrm{ML}$ 385 in a sheet-induction medium for 7 days, and qRTPCR analysis showed that $10 \mu \mathrm{M}$ ML 385 could significantly inhibit Nrf2/ARE signaling pathway of KAT6Aoverexpressed OBMSCs (Fig. 5a). Thus, $10 \mu \mathrm{M}$ ML 385 was used in our study. After sheet formation, sheets were filled in the alveolar bone defects. After 6 weeks, micro-CT was performed to observe the indexes of bone mineral density (BMD), bone volume/total volume (BV/ TV), trabecular thickness (Tb.Th), and trabecular separation (Tb.Sp). The results showed that BMD, BV/TV, $\mathrm{Tb} . \mathrm{Th}$, and Tb.Sp were all improved in KAT6Aoverexpressed OBMSC groups compared to those in the vector-overexpressed OBMSC group (Fig. 5b-f). However, compared to KAT6A-overexpressed OBMSCs, these indexes in KAT6A-overexpressed followed by ML385 treatment OBMSC group were decreased, suggesting that KAT6A promoted osteogenesis of OBMSCs through Nrf2/ARE signaling pathway.

\section{Discussion}

In our study, the relationship between KAT6A and Nrf2/ARE signaling pathway in the aging process of BMSCs was uncovered, which has not been recognized previously. As summarized in Fig. 6, our study firstly found that KAT6A was downregulated in the aging process of BMSCs, and demonstrated that KAT6A downregulation in OBMSCs could result in Nrf2/ARE signaling pathway inhibition and ROS accumulation, which further led to stemness impairment.

Osteoporosis is one important manifestation of aging, and many studies have shed light on the decreased stemness of BMSCs and proved that multifarious mechanisms including signaling pathways including $\mathrm{NAD}^{+}$ Sirt1 [26] and Notch [27], miRNA [28, 29], and organelle dysfunction [30] contributed to the decline during cellular senescence. Epigenetic alteration is one primary hallmark of aging, and the progressive accumulation of epigenetic alteration could lead to stem cell senescence through changing genomic instability and gene expression profiles. Histone acetylation, which is determined by HATs and histone deacetylases (HDACs), is an important part of aging-related epigenetic alteration, and numerous studies have shown that histone acetylation modification could affect stem cell senescence. Some studies demonstrated that HDAC inhibitor (HDACi) could improve aging-related phenotype by increasing histone acetylation level [31-33], while others found that HDACi could induce cellular senescence [34, 35]. The nonspecificity of the drugs and the specificity of different HATs and HDACs may partly account for this. Therefore, locating the key histone acetylation-related enzymes in the aging process is of great significance. In our study, decreased acetylation levels of lysine and downregulated histone $\mathrm{H} 3$ acetylation in OBMSCs were found, which suggested that histone $\mathrm{H} 3$ acetylation may play an important role in the decreased stemness of OBMSCs. The KAT6A gene, which was firstly discovered in $\mathrm{t}(8 ; 16)(\mathrm{p} 11 ; \mathrm{p} 13)$ chromosome translocations, can specifically acetylate histone H3. KAT6A has been widely known for its contribution in leukemogenesis due to its histone acetyltransferase activity [36]. And beyond that, KAT6A could also regulate Runx2, an osteogenic transcriptional factor [37], and promote the osteogenic differentiation of periodontal ligament stem cell [22]. Recent research showed that inhibition of KAT6A activity could induce the senescence of mouse embryo fibroblasts through p16INK4A-p19ARF pathway [10], indicating the potential effect of KAT6A on cellular senescence. In our study, KAT6A was found to be downregulated. Through gain- and loss-of-function experiments, decreased KAT6A expression was proven to have resulted in the decreased stemness of OBMSCs.

ROS generated from normal metabolism or external stimulus can accumulate during the aging process. They cause damage to DNA, protein, and organelle [15] and perturb cell proliferation [38], induce apoptosis [39], and impair differentiation [40]. Nrf2/ARE signaling pathway is responsible for oxygen radical scavenging, and Nrf2 deficiency can disrupt bone metabolism as Nrf2-/- mice exhibited increased bone resorption and reduced bone formation [41]. Numerous studies have confirmed disruption of Nrf2/ARE 


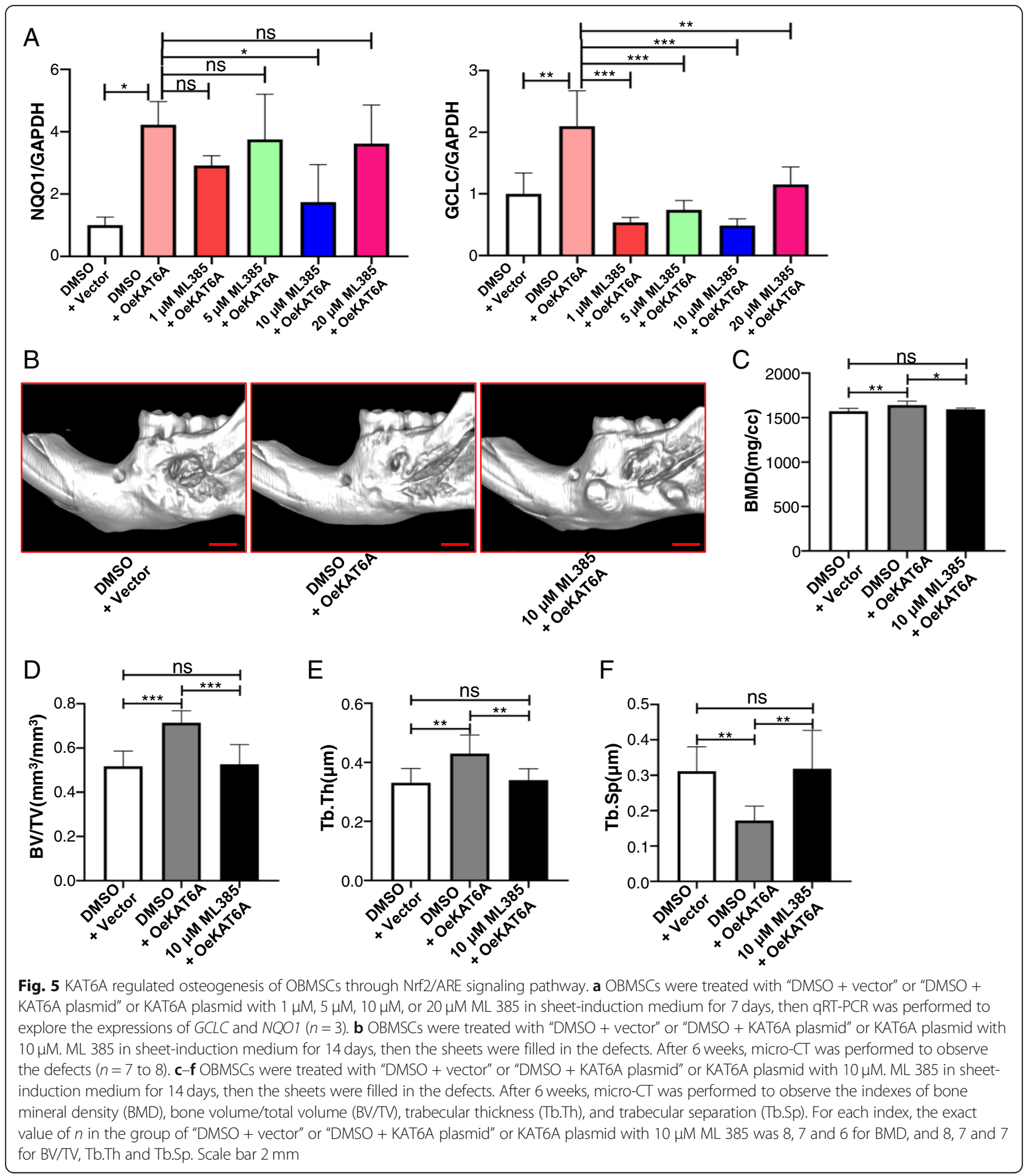

signaling pathway during the aging process $[15,42]$. One study reported that cellular localization of Nrf2 was changed in replicative senescence, and increasing nuclear Nrf2 could improve stemness of late-passage BMSCs [43]. Another study found that impaired activity of Nrf2/ARE signaling pathway was a driver mechanism in Hutchinson-Gilford progeria syndrome through high-throughput RNAi screening, and Oltipraz, a Nrf2-activating agents, could ameliorate the progeroid phenotype [42]. The connection between HATs and Nrf2/ARE signaling pathway has been reported: One 


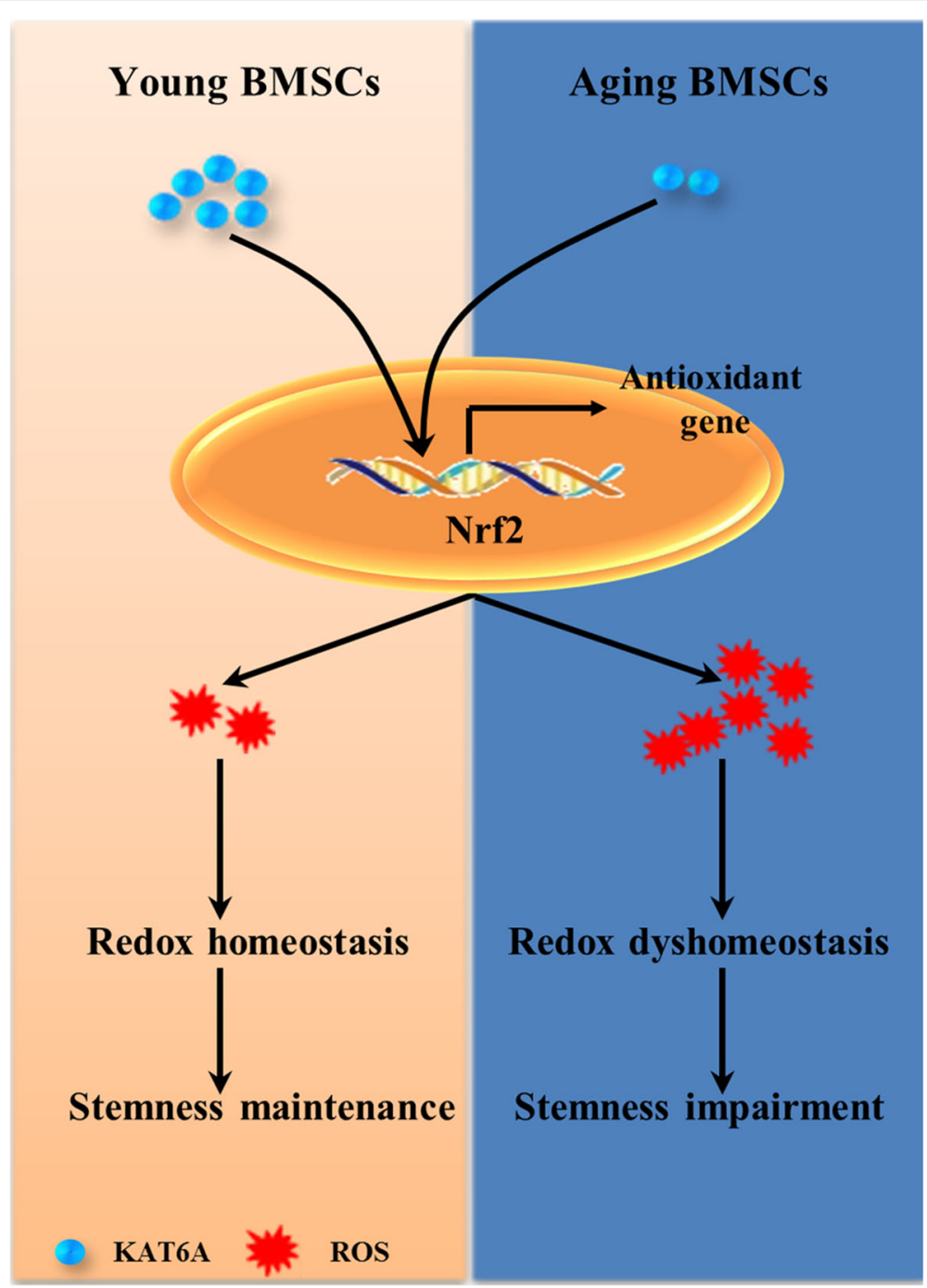

Fig. 6 Graphic summary shows the mechanism of decreased KAT6A-mediated stemness impairment in aging BMSCs. KAT6A was downregulated in aging BMSCs; this downregulation resulted in Nrf2/ARE signaling pathway inhibition and ROS accumulation, which further led to stemness impairment

study showed that coactivator P300 can regulate sodium butyrate-mediated transcriptional activation of $\mathrm{Nrf} 2$ by binding to the Nrf2 gene promoter region [44]; another study found that histone acetyltransferase MOF could increase nuclear retention of Nrf2 in $293 \mathrm{~T}$ cells and improve antioxidative responses [21]. Given these, KAT6A was speculated to regulate stemness through Nrf2/ARE signaling pathway. Firstly, KAT6A was demonstrated to regulate Nrf2/ARE signaling pathway. Further study showed that inhibited Nrf2/ ARE signaling pathway contributed to the decreased stemness of OBMSCs and KAT6A could improve stemness through the Nrf2/ARE signaling pathway. What is more, in vivo study was also performed to investigate whether KAT6A-mediated Nrf2/ARE signaling pathway could regulate bone formation of OBMSCs, an index reflecting the stemness of OBMSCs. Results showed that upregulated KAT6A in OBMSCs could promote the osteogenesis ability of OBMSCs, and inhibition of Nrf2/ARE signaling pathway could reverse KAT6A-mediated improvement of osteogenesis ability of OBMSCs.

\section{Conclusions}

This study confirmed that aging BMSCs lost stemness compared to YBMSCs and demonstrated KAT6A-mediated decreased stemness of aging BMSCs and clarified the mechanism of KAT6A by regulating the Nrf2/ARE signaling pathway. With respect to research significance, this study not only finds a new mechanism for the decreased stemness of aging BMSCs but also provides a feasible strategy for the treatment of age-related osteoporosis. 


\section{Supplementary Information}

The online version contains supplementary material available at https://doi. org/10.1186/s13287-021-02164-5.

Additional file 1: Table S1. Sequence of primers for qRT-PCR.

Additional file 2: Figure S1. The stemness of OBMSCs was decreased compared to YBMSCs. (a) CCK-8 was performed to explore the proliferative capacity of YBMSCs and OBMSCs $(n=3)$. (b) The colony-forming abilities of YBMSCs and OBMSCs were explored by crystal violet after culture for 12 days $(n=3)$. (c) After osteogenic induction for 14 days, expressions of osteogenic-related genes of Funx2, OCN and BMP2 were detected in YBMSCs and OB < SCs ( $n=3$ ). (d) After osteogenic induction for 21 days, alizarin red staining was performed in YBMSCs and OBMSCs $(n=3)$. ${ }^{*} P<$ 0.05 ; ${ }^{* *} P<0.01 ;{ }^{* * *} P<0.001$.

\section{Abbreviations}

BMSCs: Bone marrow-derived mesenchymal stem cells; YBMSCs: BMSCs from the young; OBMSCs: BMSCs from the old; HATs: Histone acetyltransferases; Runx2: RUNX family transcription factor 2; ROS: Reactive oxygen species; Nrf2: Nuclear factor erythroid 2-related factor 2; CFU-F: Colony-forming unitfibroblastic; CCK-8: Cell Counting Kit-8; qRT-PCR: Quantitative reverse transcription polymerase chain reaction; OCN: Osteocalcin; BMP2: Bone morphogenetic protein 2; GAPDH: Glyceraldehyde-3-phosphate dehydrogenase; HDACs: Histone deacetylases; HDACi: HDAC inhibitor

\section{Acknowledgements}

Not applicable

\section{Authors' contributions}

WQT performed the design of the work and provided financial aid; FDD performed this study and was a major contributor in writing the manuscript; WYZ performed western blot analysis of Nrf2 protein and GRT-PCR analysis; ZQM performed animal studies and results analysis; LB performed the design of the work; ZXG, ZY, and WY helped BMSC collection and data analysis. All authors read and approved the final manuscript.

\section{Funding}

This work was supported by the grants from the National Natural Science Foundation of China (Nos. 81771069).

\section{Availability of data and materials}

The datasets used and/or analyzed during the current study are available from the corresponding author on reasonable request.

\section{Ethics approval and consent to participate}

Human BMSCs collected from the First Hospital Affiliated to the Fourth Military Medical University were used in this study, and these cells were collected from informed, consenting persons. Their use has been approved by the Ethics Committee of Fourth Military Medical University. The animal study follows the committee guidelines of Animal Care Committee of Fourth Military Medical University, and the experimental protocol about animals was approved.

\section{Consent for publication}

Not applicable

\section{Competing interests}

The authors declare that they have no competing interests.

Received: 3 November 2020 Accepted: 12 January 2021

Published online: 04 February 2021

\section{References}

1. Ma Y, Qi M, An Y, Zhang L, Yang R, Doro DH, et al. Autophagy controls mesenchymal stem cell properties and senescence during bone aging. Aging Cell. 2018;17(1):e12709.

2. Marie PJ. Targeting integrins to promote bone formation and repair. Nat Rev Endocrinol. 2013;9(5):288-95.

3. Hou Y, Dan X, Babbar M, Wei Y, Hasselbalch SG, Croteau DL, et al. Ageing as a risk factor for neurodegenerative disease. Nat Rev Neurol. 2019;15(10):565-81.
4. Zhang W, Qu J, Liu GH, Belmonte J. The ageing epigenome and its rejuvenation. Nat Rev Mol Cell Biol. 2020;21(3):137-50.

5. Roth SY, Denu JM, Allis CD. Histone acetyltransferases. Annu Rev Biochem. 2001;70:81-120.

6. Xue P, Li B, An Y, Sun J, He X, Hou R, et al. Decreased MORF leads to prolonged endoplasmic reticulum stress in periodontitis-associated chronic inflammation. Cell Death Differ. 2016;23(11):1862-72.

7. Zhang $P$, Liu $Y$, Jin $C$, Zhang M, Tang F, Zhou Y. Histone acetyltransferase GCN5 regulates osteogenic differentiation of mesenchymal stem cells by inhibiting NF-kappaB. J Bone Miner Res. 2016;31(2):391-402.

8. Jing H, Su X, Gao B, Shuai Y, Chen J, Deng Z, et al. Epigenetic inhibition of Wnt pathway suppresses osteogenic differentiation of BMSCs during osteoporosis. Cell Death Dis. 2018;9(2):176.

9. Sheikh BN, Yang Y, Schreuder J, Nilsson SK, Bilardi R, Carotta S, et al. MOZ (KAT6A) is essential for the maintenance of classically defined adult hematopoietic stem cells. BLOOD. 2016;128(19):2307-18.

10. Baell JB, Leaver DJ, Hermans SJ, Kelly GL, Brennan MS, Downer NL, et al. Inhibitors of histone acetyltransferases KAT6A/B induce senescence and arrest tumour growth. Nature. 2018:560(7717):253-7.

11. Sheikh BN, Phipson B, El-Saafin F, Vanyai HK, Downer NL, Bird MJ, et al. MOZ (MYST3, KAT6A) inhibits senescence via the INK4A-ARF pathway. Oncogene. 2015; 34(47):5807-20

12. Rokudai S, Laptenko O, Arnal SM, Taya Y, Kitabayashi I, Prives C. MOZ increases p53 acetylation and premature senescence through its complex formation with PML. Proc Natl Acad Sci U S A. 2013;110(10):3895-900.

13. Butterfield DA, Halliwell B. Oxidative stress, dysfunctional glucose metabolism and Alzheimer disease. Nat Rev Neurosci. 2019;20(3):148-60.

14. Camici GG, Savarese G, Akhmedov A, Luscher TF. Molecular mechanism of endothelial and vascular aging: implications for cardiovascular disease. Eur Heart J. 2015:36(48):3392-403.

15. Chen F, Liu Y, Wong NK, Xiao J, So KF. Oxidative stress in stem cell aging. Cell Transplant. 2017;26(9):1483-95.

16. Cuadrado A, Rojo Al, Wells G, Hayes JD, Cousin SP, Rumsey WL, et al. Therapeutic targeting of the NRF2 and KEAP1 partnership in chronic diseases. Nat Rev Drug Discov. 2019;18(4):295-317.

17. Shelar SB, Narasimhan M, Shanmugam G, Litovsky SH, Gounder SS, Karan G, et al. Disruption of nuclear factor (erythroid-derived-2)-like 2 antioxidant signaling: a mechanism for impaired activation of stem cells and delayed regeneration of skeletal muscle. FASEB J. 2016;30(5):1865-79.

18. Vono R, Jover GE, Spinetti G, Madeddu P. Oxidative stress in mesenchymal stem cell senescence: regulation by coding and noncoding RNAs. Antioxid Redox Signal. 2018;29(9):864-79.

19. Gao L, Yuan F, Che G, Xiao X, Nie X, Wang Y, et al. Epigenetic modifications but not genetic polymorphisms regulate KEAP1 expression in colorectal cancer. J Cell Biochem. 2019;120(8):12311-20.

20. Guo Y, Yu S, Zhang C, Kong AN. Epigenetic regulation of Keap1-Nrf2 signaling. Free Radic Biol Med. 2015;88(Pt B):337-49.

21. Chen Z, Ye X, Tang N, Shen S, Li Z, Niu X, et al. The histone acetylranseferase hMOF acetylates Nrf2 and regulates anti-drug responses in human non-small cell lung cancer. Br J Pharmacol. 2014;171(13):3196-211.

22. Sun J, Dong Z, Zhang $Y$, He X, Fei $D$, Jin F, et al. Osthole improves function of periodontitis periodontal ligament stem cells via epigenetic modification in cell sheets engineering. Sci Rep. 2017:7(1):5254.

23. Doyon Y, Cayrou C, Ullah M, Landry AJ, Cote V, Selleck W, et al. ING tumor suppressor proteins are critical regulators of chromatin acetylation required for genome expression and perpetuation. Mol Cell. 2006;21(1):51-64.

24. Qiu Y, Wan B, Liu G, Wu Y, Chen D, Lu M, et al. Nrf2 protects against seawater drowning-induced acute lung injury via inhibiting ferroptosis. Respir Res. 2020;21(1):232.

25. Chen $X$, Yang JH, Cho SS, Kim JH, Xu J, Seo K, et al. 5-Caffeoylquinic acid ameliorates oxidative stress-mediated cell death via Nrf2 activation in hepatocytes. Pharm Biol. 2020;58(1):999-1005.

26. Pi C, Yang Y, Sun Y, Wang H, Sun $H$, Ma M, et al. Nicotinamide phosphoribosyltransferase postpones rat bone marrow mesenchymal stem cell senescence by mediating NAD(+ )-Sirt1 signaling. Aging (Allbany NY). 2019;11(11):3505-22.

27. Tian $Y, X u Y, X u e ~ T$, Chen $L$, Shi B, Shu B, et al. Notch activation enhances mesenchymal stem cell sheet osteogenic potential by inhibition of cellular senescence. Cell Death Dis. 2017:8(2):e2595.

28. Xu R, Shen X, Si Y, Fu Y, Zhu W, Xiao T, et al. MicroRNA-31a-5p from aging BMSCs links bone formation and resorption in the aged bone marrow microenvironment. Aging Cell. 2018;17(4):e12794. 
29. Yu KR, Lee S, Jung JW, Hong IS, Kim HS, Seo Y, et al. MicroRNA-141-3p plays a role in human mesenchymal stem cell aging by directly targeting ZMPSTE24. J Cell Sci. 2013;126(Pt 23):5422-31.

30. Lv YJ, Yang Y, Sui BD, Hu CH, Zhao P, Liao L, et al. Resveratrol counteracts bone loss via mitofilin-mediated osteogenic improvement of mesenchymal stem cells in senescence-accelerated mice. Theranostics. 2018:8(9):2387-406.

31. Wang Y, Chen T, Yan H, Qi H, Deng C, Ye T, et al. Role of histone deacetylase inhibitors in the aging of human umbilical cord mesenchymal stem cells. J Cell Biochem. 2013;114(10):2231-9.

32. Lee AR, Kishigami S, Amano T, Matsumoto K, Wakayama T, Hosoi Y. Nicotinamide: a class III HDACi delays in vitro aging of mouse oocytes. J Reprod Dev. 2013;59(3):238-44.

33. Walsh ME, Bhattacharya A, Sataranatarajan K, Qaisar R, Sloane L, Rahman MM, et al. The histone deacetylase inhibitor butyrate improves metabolism and reduces muscle atrophy during aging. Aging Cell. 2015:14(6):957-70.

34. Tapia PC. Histone-deacetylase inhibitors may accelerate the aging process in stem cell-dependent mammals: stem cells, Ku70, and Drosophila at the crossroads. Med Hypotheses. 2006;66(2):332-6.

35. Jung JW, Lee S, Seo MS, Park SB, Kurtz A, Kang SK, et al. Histone deacetylase controls adult stem cell aging by balancing the expression of polycomb genes and jumonji domain containing 3. Cell Mol Life Sci. 2010;67(7):1165-76.

36. Deguchi K, Ayton PM, Carapeti M, Kutok JL, Snyder CS, Williams IR, et al. MOZ-TIF2-induced acute myeloid leukemia requires the MOZ nucleosome binding motif and TIF2-mediated recruitment of CBP. Cancer Cell. 2003;3(3): 259-71.

37. Pelletier N, Champagne N, Stifani S, Yang XJ. MOZ and MORF histone acetyltransferases interact with the Runt-domain transcription factor Runx2. Oncogene. 2002;21(17):2729-40.

38. latsenko I, Boquete JP, Lemaitre B. Microbiota-derived lactate activates production of reactive oxygen species by the intestinal NADPH oxidase Nox and shortens Drosophila lifespan. Immunity. 2018;49(5):929-42.

39. Rouault-Pierre K, Lopez-Onieva L, Foster K, Anjos-Afonso F, Lamrissi-Garcia I, Serrano-Sanchez M, et al. HIF-2alpha protects human hematopoietic stem/ progenitors and acute myeloid leukemic cells from apoptosis induced by endoplasmic reticulum stress. Cell Stem Cell. 2013;13(5):549-63.

40. Khacho M, Clark A, Svoboda DS, Azzi J, MacLaurin JG, Meghaizel C, et al. Mitochondrial dynamics impacts stem cell identity and fate decisions by regulating a nuclear transcriptional program. Cell Stem Cell. 2016;19(2):232-47.

41. Sun YX, Xu AH, Yang Y, Li J. Role of Nrf2 in bone metabolism. J Biomed Sci. 2015;22:101

42. Kubben N, Zhang W, Wang L, Voss TC, Yang J, Qu J, et al. Repression of the antioxidant NRF2 pathway in premature aging. Cell. 2016;165(6):1361-74.

43. Yoon DS, Choi Y, Lee JW. Cellular localization of NRF2 determines the selfrenewal and osteogenic differentiation potential of human MSCs via the P53-SIRT1 axis. Cell Death Dis. 2016;7:e2093.

44. Wu J, Jiang Z, Zhang $H$, Liang W, Huang W, Zhang $H$, et al. Sodium butyrate attenuates diabetes-induced aortic endothelial dysfunction via P300mediated transcriptional activation of Nrf2. Free Radic Biol Med. 2018;124: 454-65.

\section{Publisher's Note}

Springer Nature remains neutral with regard to jurisdictional claims in published maps and institutional affiliations.

Ready to submit your research? Choose BMC and benefit from:

- fast, convenient online submission

- thorough peer review by experienced researchers in your field

- rapid publication on acceptance

- support for research data, including large and complex data types

- gold Open Access which fosters wider collaboration and increased citations

- maximum visibility for your research: over $100 \mathrm{M}$ website views per year

At $\mathrm{BMC}$, research is always in progress.

Learn more biomedcentral.com/submissions 